\title{
Combined inhibition of atypical PKC and histone deacetylase 1 is cooperative in basal cell carcinoma treatment
}

Amar N. Mirza, ${ }^{1}$ Micah A. Fry, ${ }^{2}$ Nicole M. Urman, ${ }^{1}$ Scott X. Atwood, ${ }^{1}$ Jon Roffey, ${ }^{3}$ Gregory R. Ott, ${ }^{4}$ Bin Chen, ${ }^{5}$ Alex Lee, ${ }^{2}$ Alexander S. Brown, ${ }^{1}$ Sumaira Z. Aasi, ${ }^{1}$ Tyler Hollmig, ${ }^{1}$ Mark A. Ator, ${ }^{4}$ Bruce D. Dorsey, ${ }^{4}$ Bruce R. Ruggeri, ${ }^{4}$ Craig A. Zificsak, ${ }^{4}$ Marina Sirota, ${ }^{5}$ Jean Y. Tang,, 2 Atul Butte, ${ }^{5}$ Ervin Epstein, ${ }^{2}$ Kavita Y. Sarin, ${ }^{1}$ and Anthony E. Oro ${ }^{1}$

'Program in Epithelial Biology and Department of Dermatology, Stanford University School of Medicine, Stanford, California, USA. ${ }^{2}$ Children's Hospital Oakland Research Institute, Oakland, California, USA. ${ }^{3}$ CRUK Therapeutic Discovery Laboratories, London Bioscience Innovation Centre, London, United Kingdom. ${ }^{4}$ Teva Branded Pharmaceutical Products R\&D, West Chester, Pennsylvania, USA. Institute for Computational Health Sciences, UCSF, San Francisco, California, USA.

Advanced basal cell carcinomas (BCCs) circumvent Smoothened (SMO) inhibition by activating CLI transcription factors to sustain the high levels of Hedgehog $(\mathrm{HH})$ signaling required for their survival. Unfortunately, there is a lack of efficacious therapies. We performed a gene expressionbased drug repositioning screen in silico and identified the FDA-approved histone deacetylase (HDAC) inhibitor, vorinostat, as a top therapeutic candidate. We show that vorinostat only inhibits proliferation of BCC cells in vitro and BCC allografts in vivo at high dose, limiting its usefulness as a monotherapy. We leveraged this in silico approach to identify drug combinations that increase the therapeutic window of vorinostat and identified atypical PKC $\mathrm{V} / \mathrm{K}$ (aPKC) as a HDAC costimulator of HH signaling. We found that aPKC promotes GLI1-HDAC1 association in vitro, linking two positive feedback loops. Combination targeting of HDAC1 and aPKC robustly inhibited CLI1, lowering drug doses needed in vitro, in vivo, and ex vivo in patient-derived BCC explants. We identified a bioavailable and selective small-molecule aPKC inhibitor, bringing the pharmacological blockade of aPKC and HDAC1 into the realm of clinical possibility. Our findings provide a compelling rationale and candidate drugs for combined targeting of HDAC1 and aPKC in HH-dependent cancers.

Authorship note: K.Y. Sarin and A.E. Oro are co-senior authors.

Conflict of interest: A.E. Oro receives clinical investigation funds from Novaris. J.Y. Tang receives equity from PellePharm. $\mathrm{R}$ is named as an inventor on a patent (patent number W0/2014/052699 A1) that describes development of CRT0329868.

Submitted: August 23, 2017 Accepted: September 29, 2017 Published: November 2, 2017

\section{Reference information:}

JCI Insight. 2017;2(21):e97071. https://doi.org/10.1172/jici. insight.97071.

\section{Introduction}

Basal cell carcinomas (BCCs) require high levels of Hedgehog $(\mathrm{HH})$ signaling for survival and growth (1-4). Activation of the $\mathrm{HH}$ pathway involves the $\mathrm{HH}$ ligand binding to Patched-1, thereby relieving inhibition of Smoothened (SMO). This results in activation of the GLI family of transcription factors, which ultimately promote transcription of $\mathrm{HH}$ target genes, including Gli1 itself. SMO inhibitors have recently been FDA approved for BCC treatment, but drug resistance has emerged as a significant problem $(1,2,5)$. As BCCs uniformly depend on the $\mathrm{HH}$ pathway for growth (2), resistant BCCs evolve to circumvent pharmacological blockade at the level of SMO using pathway-intrinsic mutations as well as noncanonical mechanisms of GLI activation $(2,3)$.

Recently, we identified atypical PKC $1 / K(\mathrm{aPKC})$ overactivation as a powerful mechanism of drug resistance in BCC (3). aPKC phosphorylation of the GLI1 zinc-finger domain results in chromatin association, gene transcription, and HH pathway activation downstream of inputs from SMO and Patched-1. Furthermore, GLI promotes transcription of aPKC, forming another positive feedback loop with GLI. Overactivation of this noncanonical $\mathrm{HH}$ signaling pathway drives pathway activation and vismodegib escape in advanced BCC (3). Small-molecule inhibitors of aPKC, allosteric (6) or orthosteric (7), are in development but have not been applied to treat BCC.

GLI proteins are further regulated, downstream of SMO, through acetylation by p300 and subsequent deacetylation. The deacetylation of GLI1/2 at K518 and K757, respectively, by histone deacetylase $1 / 2$ $(\mathrm{HDAC} 1 / 2)$ is a critical step in the nuclear maturation process of GLI transcription factors required for chro- 
matin association and gene transcription (8). HDAC1 is itself a transcriptional target of GLI, creating a third positive feedback loop of $\mathrm{HH}$ signaling. Of particular interest, HDAC inhibition has been proposed for the treatment of many $\mathrm{HH}$-driven cancers (8-11). HDAC inhibitors block growth and promote apoptosis by altering the histone-DNA complex and by altering the acetylation status of nonhistone proteins (12). Vorinostat, a class I/II HDAC inhibitor, is currently FDA approved for the treatment of cutaneous lymphoma (13). Unfortunately, HDAC inhibition has been hampered by its broadly cytotoxic nature.

De novo drug discovery remains challenging due to the lack of validated targets and the cost of clinical development (14). To address this problem, we utilized an in silico drug repositioning approach to identify existing FDA-approved drugs for applications based on gene expression profiles (15). This approach has been successfully used to predict novel therapeutics for lung cancer (16), Crohn's disease (17), and, most recently, dermatomyositis (18).

Here, we utilized this repositioning approach for BCC and identify HDAC inhibition as a powerful therapy for BCC in vitro and in vivo. We found that effectively halting GLI deacetylation via vorinostat alone is clinically unachievable. To address this shortcoming, we further characterized the mechanism of GLI1-HDAC1 interaction. We found that aPKC activity recruits HDAC1 to GLI1, linking two powerful positive feedback loops in the $\mathrm{HH}$ pathway. We found that combined therapy targeting both HDAC1 and aPKC halts the nuclear maturation of GLI1 and antagonizes BCCs at lower doses than either drug alone. However, this therapeutic strategy would remain irrelevant without the development of a clinically useful small-molecule inhibitor of aPKC. We identify an ATP-competitive aPKC small-molecule inhibitor that demonstrates an order of magnitude of improvement in potency over previous generation orthosteric (7) and allosteric (6) inhibitors, with high bioavailability and efficacy in BCC. We demonstrate that the use of this compound along with vorinostat can effectively disrupt the aPKC-HDAC1 axis in BCC.

\section{Results}

Drug repositioning identifies vorinostat for BCC treatment. The RNA expression patterns of early (19) and advanced (6) BCCs were compared with normal skin samples to generate a core set of genes altered in early-stage and advanced BCCs, respectively (Figure 1A; Supplemental Methods; Supplemental Figures 1 and 2; and Supplemental Tables 1 and 2; supplemental material available online with this article; https://doi.org/10.1172/ jci.insight.97071DS1). The resulting BCC signatures were integrated with gene expression data from over 1,100 FDA-approved drugs contained in the Library of Integrated Cellular Signature (LINCS) to identify compounds that reverse the disease gene expression signature. Predicted therapeutics were identified independently for advanced and early-stage BCCs (Supplemental Tables 3 and 4 and Supplemental Figures 3 and 4) and subsequently intersected to predict therapeutics with efficacy for both early-stage and advanced BCCs (Supplemental Figure 5). This approach identified the HDAC inhibitor, vorinostat, as a top candidate therapy (Figure 1, A-C, and Supplemental Figure 5). Vorinostat and two other drug candidates, rosiglitazone and tacrolimus, were screened for their ability to suppress the growth of BCC allografts obtained from a murine

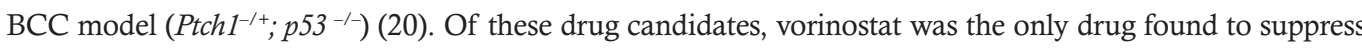
BCC tumor growth in vivo (Supplemental Figure 6).

To study the efficacy of HDAC inhibition on BCCs in vitro, a murine BCC cell line (ASZ) (21) resistant to SMO inhibitors but dependent on GLI1 was treated with increasing doses of vorinostat, a class-I/ II HDAC inhibitor, and parthenolide, a HDAC1-specific inhibitor (22). Vorinostat treatment resulted in a dose-dependent reduction in HH signaling, as assayed by qPCR of Gli1 mRNA, a canonical target gene of the HH pathway (Figure 1D), and by GLI1 protein (Supplemental Figure 7). Similarly, parthenolide produced a dose-dependent reduction in Gli1 mRNA (Figure 1D). To determine which HDACs mediate the GLI1 suppression by vorinostat, we assayed target gene transcription across a panel of HDAC inhibitors over a 1-hour treatment window. All HDAC1/2 inhibitors produced significant reductions in target gene transcription, while HDAC3-specific inhibitor RGFP966 had no effect on HH output (Figure 1E). To specifically target HDAC1/2, we performed genetic knockdown against HDAC1 and HDAC2 by siRNA and observed strong inhibition of pathway output (Supplemental Figure 8). Taken together, these data indicate that vorinostat inhibits $\mathrm{HH}$ signaling primarily through its repression of HDAC1/2 activity.

HDAC inhibitor treatment resulted in significant BCC cell death within 48 hours (Figure 1, F and $\mathrm{G})$. However, the doses required to achieve robust $\mathrm{HH}$ inhibition and cell death were substantially higher than the reported $\mathrm{IC}_{50}$ of the drugs, resulting in toxicity to normal keratinocytes at the same doses (Supplemental Figure 9). Similarly, vorinostat treatment administered in vivo on BCC allografts 
A

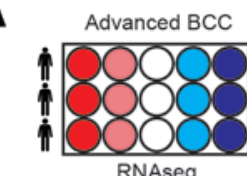

6 Advanced BCC vs 5 Normal Skin

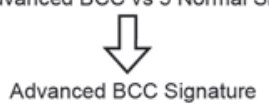

79 up, 59 down
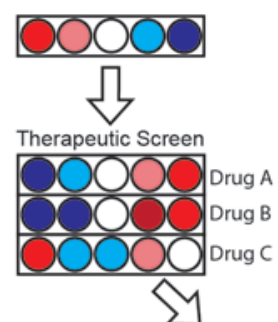

Common predicted therapeutics

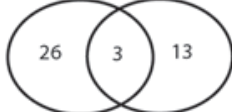

Advanced BCC Early BCC

D

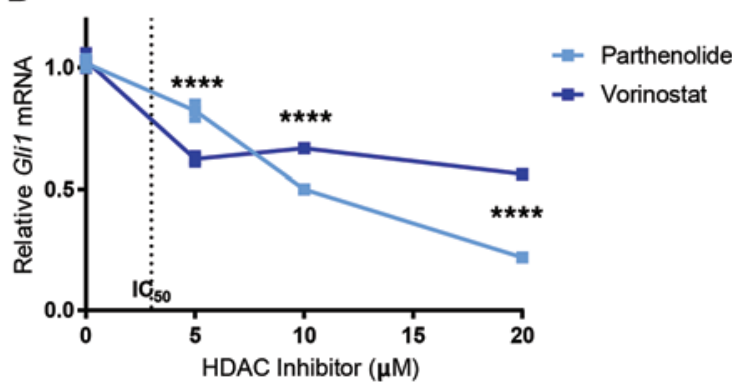

Early BCC

23 BCC vs 8 Normal Skin

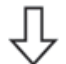

Early BCC Signature 450 up, 574 down

几

Therapeutic Screen

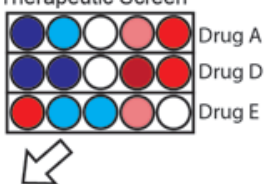

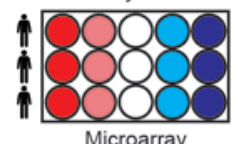

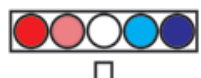

ASZ

$\mathbf{F}$

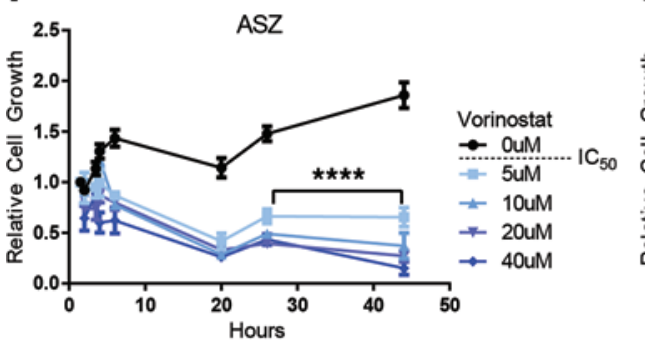

H

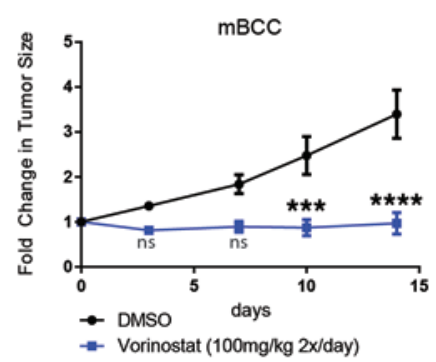

B

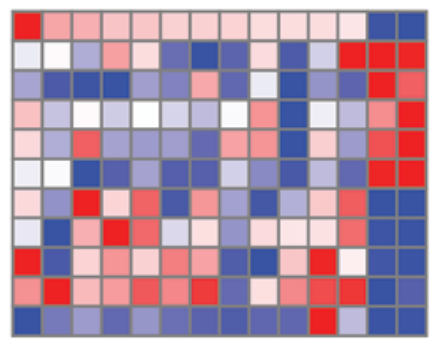

$\mathrm{BCC}$

vorinostat

troglitazone

albendazole

bumetanide

hydrocortisone

tretinoin

ranolazine

quinine

fulvestrant

carbenoxolone
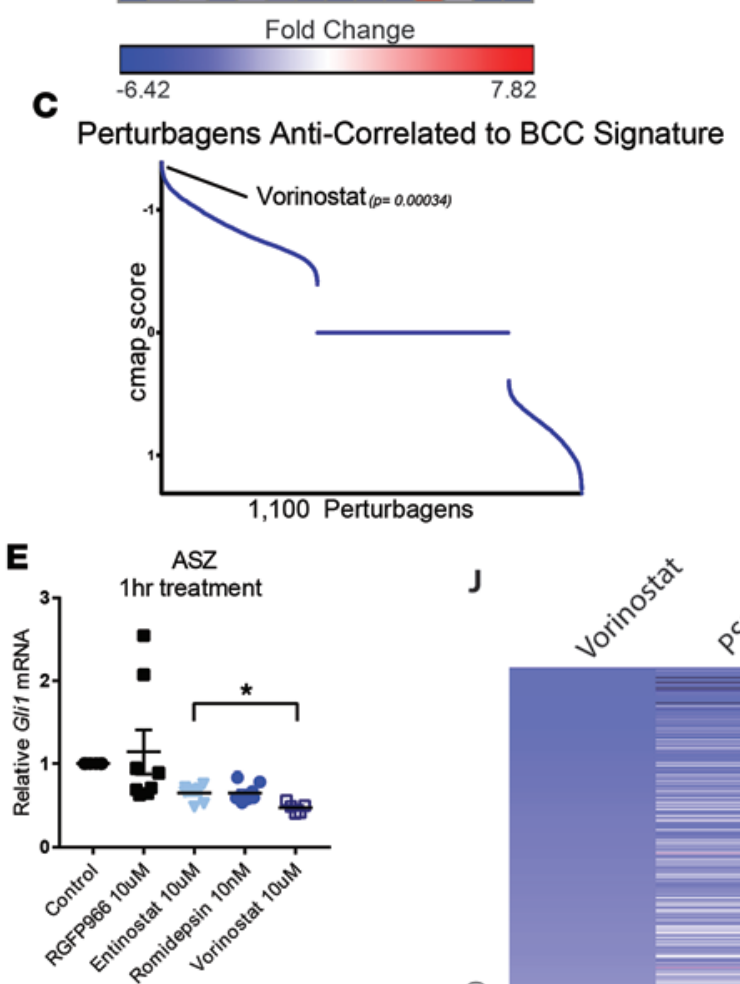

HDAC Inhibitors
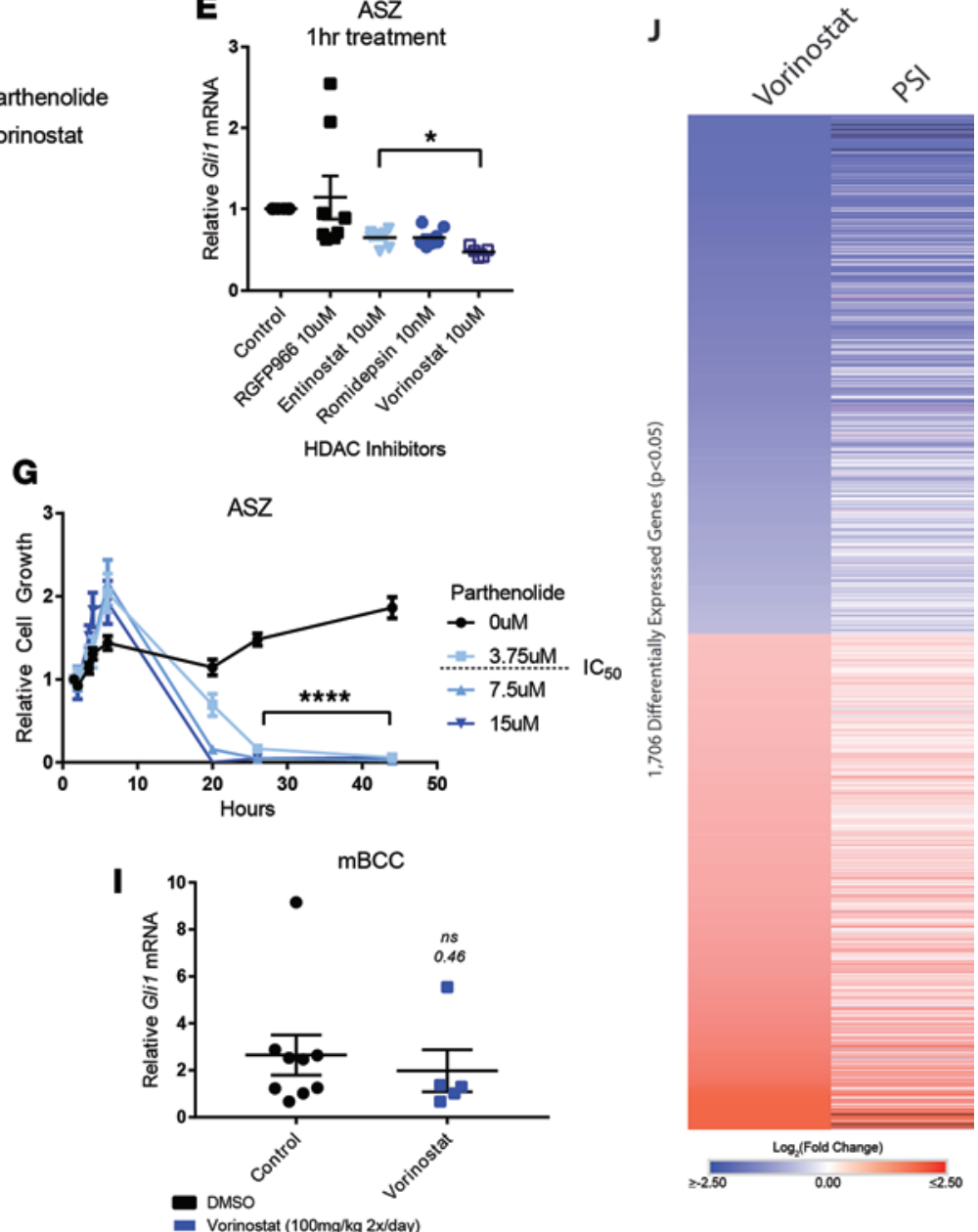

stat $(100 \mathrm{mg} / \mathrm{kg} 2 \times / \mathrm{da}$

Figure 1. HDAC inhibition suppresses BCC growth in vitro and in vivo. (A) Schematic of generation of BCC expression signature, followed by drug-repositioning analysis using LINCS. (B) Heatmap of expression changes in landmark genes in advanced BCC and expression changes following drug treatment. Select perturbagens are depicted. (C) Ranked anticorrelation score (cmap score) of all assay perturbagens following LINCS analysis ranked by score. HH target gene transcript dose response to vorinostat and parthenolide (D) in ASZ cells with vorinostat I $\mathrm{C}_{50}$, represented by dotted line ( $n=9$; ANOVA), or across a panel of HDAC1/2 targeting inhibitors (blue) or HDAC3 (gray) over an acute time course $(n=9$; ANOVA) (E). Cli1 transcript measured by qRT-PCR 
and normalized against HPRT1. Relative ASZ cell growth measured by Real-Time Glo Reagent following vorinostat (F) and parthenolide (G) treatment ( $n$ = 3 per time point; 2-way ANOVA; significance is only illustrated for $t>20$ hours for clarity). (H) Tumor size measurements following vorinostat treatment of mouse BCCs and (I) Gli1 mRNA measured by qPCR ( $n=6$; 2-way ANOVA and Student's $t$ test, respectively). All control measurements are black, while HDAC inhibitor treatment measurements are shades of blue, varying in concentration, as indicated ( $\mathbf{F}$ and $\mathbf{G})$ or to differentiate inhibitors ( $\mathbf{D}$ and $\mathbf{E})$. (J) $\log _{2}$ fold change of normalized read counts from RNA sequencing of murine BCC allografts treated with vorinostat $(n=2)$ or PSI $(n=1)$ as compared with untreated controls $(n=2)$. Error bars represent SEM. ${ }^{*} P<0.05,{ }^{* *} P<0.001,{ }^{* * *} P<0.0001 ; P$ value calculation as in ref. 15.

showed only partial efficacy in preventing tumor progression. Twice daily administration of $100 \mathrm{mg} /$ $\mathrm{kg}$ vorinostat resulted only in tumor growth stasis (Figure $1 \mathrm{H}$ ) and only a partial decrease in Gli1 mRNA (Figure 1I; $P=0.46$ ). The requirement for such high doses of drug with only partial HH suppression limits the clinical usefulness of vorinostat for BCCs as a monoagent.

Another explanation for the partial efficacy of vorinostat in BCC is that the bulk of its effect results in non-HH-related inhibition. To determine if the in vivo change in tumor growth was a result of genomewide $\mathrm{HH}$ pathway inhibition, we performed RNA sequencing on murine BCCs that responded to treatment. We assessed gene expression changes in tumors treated with vorinostat or PSI, an aPKC inhibitor that we previously demonstrated reduces $\mathrm{HH}$ target gene transcription in BCC (3). We observe a striking overlap of significant differentially expressed genes $(P<0.05)$ between the two treatments groups, indicating that vorinostat inhibits $\mathrm{HH}$ transcriptional output genome wide (Figure $1 \mathrm{~J}$ ). Therefore we conclude that vorinostat acts through $\mathrm{HH}$ suppression in $\mathrm{BCC}$, but adequate suppression requires intolerable levels of drug.

aPKC promotes GLI1 deacetylation by HDAC1. In order to broaden the therapeutic window of vorinostat, we queried the LINCS database with drug candidates that could be used in combination with vorinostat. Because our RNA-sequencing analysis demonstrated significant similarities in gene expression changes correlated with vorinostat and PSI treatment, we went on to determine the significance of this overlap (Figure $1 \mathrm{~J}$ ). We recently identified overexpression of aPKC as a powerful mechanism of drug resistance in advanced BCCs (3). Genetic depletion of $\mathrm{aPKC}_{1}$ results in profound $\mathrm{HH}$ pathway inhibition in ASZ cells (Supplemental Figure 10). Comparing a gene set of 3,171 transcripts altered in a BCC cell line treated with PSI with data in the LINCS database, we observed a strong correlation in expression changes between aPKC inhibition and many HDAC inhibitors (Figure 2A and Supplemental Tables 5 and 6). This observation, coupled with previous data demonstrating that inhibition of $\operatorname{aPKC}(23)$ or $\operatorname{HDAC1}(8,11)$ results in an abolishment of GLI1's chromatin affinity, as assayed by ChIP without perturbing its nuclear-cytoplasmic trafficking, led us to the hypothesis that aPKC and HDAC1 work in concert to modulate GLI1's transcriptional status.

We therefore sought to investigate the epistatic relationship between aPKC and the acetylation/deacetylation machinery of GLI1 by rescue experiments. ASZ cells were transiently transfected to overexpress HDAC1 or p300. Cell viability in these two populations was measured over 24 hours to confirm consistency. Half of each population was subjected to PSI after 24 hours and observed for changes in cell viability. Overexpression of HDAC1 rescued ASZ cells from aPKC inhibition by PSI (Figure 2B and Supplemental Figure 11), while p300-overexpressing cells remained sensitive to PSI (Figure 2C and Supplemental Figure 11). These results place aPKC downstream of p300 and upstream of HDAC1 in the nuclear maturation pathway of GLI1.

Given the position of aPKC between p300 and HDAC1 and our previous studies showing that aPKC does not affect GLI protein stability or nuclear localization (3), we hypothesized that aPKC promotes GLI1 deacetylation by HDAC1 recruitment. Consistent with this model, we found that the association between GLI1 and HDAC1 depends on aPKC's activity. Pharmacological aPKC inhibition disrupted the GLI1HDAC1 interaction in the context of overexpression in HEK 293T and ASZ cells (Figure 2, E and F) or at endogenous levels in ASZ cells (Supplemental Figure 12). aPKC inhibition did not perturb the interaction of another regulator of GLI1, Suppressor of Fused ( $\mathrm{SuFu}$ ), confirming the specificity of the GLI1-HDAC1 effect (Figure 2E). Furthermore, proximity ligation assays detected an interaction between endogenous GLI1 and HDAC1, with the loss of a specific nuclear interaction upon aPKC inhibition in ASZ cells (Figure 2D). GLI1 retained nuclear localization upon aPKC inhibition (Supplemental Figure 13). These data suggest that aPKC plays an active role in recruiting HDAC1 to GLI1.

Next, we investigated if aPKC's control of the GLI1-HDAC1 interaction modulates GLI1 deacetylation. Compared with WT cells, cells with overexpression of an acetyl-deficient GLI1 (K518R), the major site for GLI1 acetylation, were resistant to aPKC inhibition (Figure 2G; $P<0.0001$ ). Conversely, aPKC inhibition resulted in GLI1 hyperacetylation, as measured by immunoblotting with an acetyl lysine antibody following FLAG-GLI1 immunoprecipitation (Figure $2 \mathrm{H}$ ). Thus, aPKC controls GLI1 acetylation by modifying HDAC1 recruitment. 
A

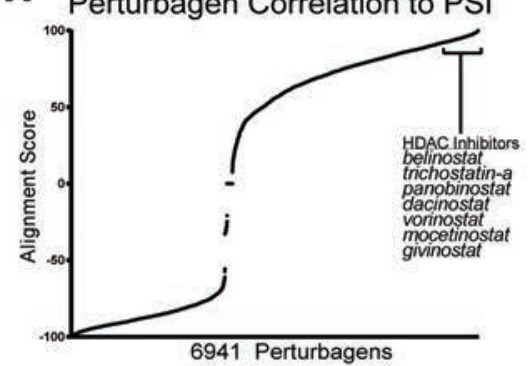

B

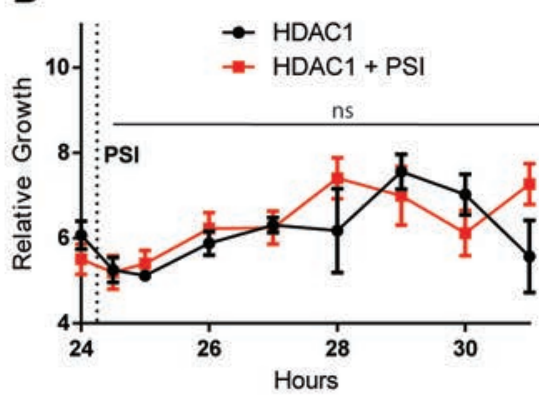

C

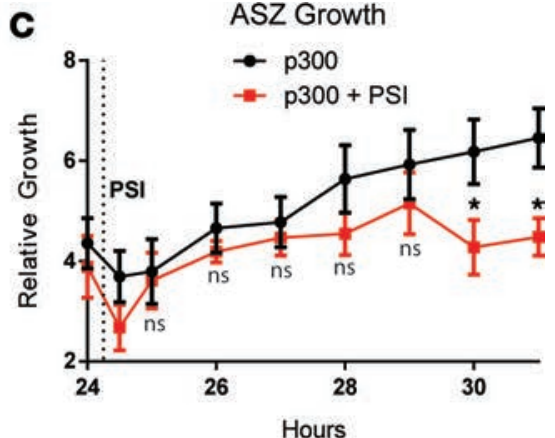

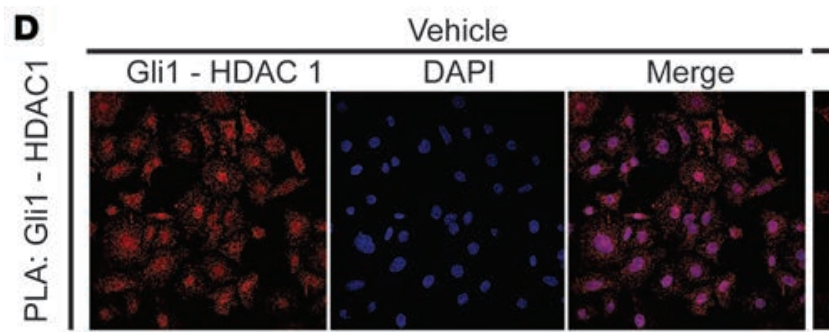

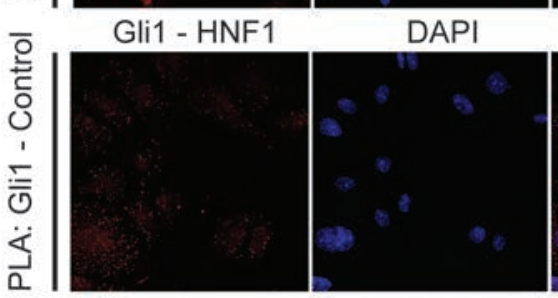

Merge

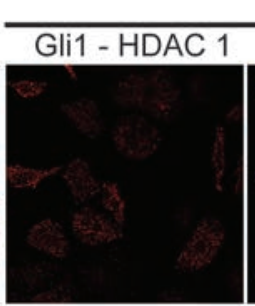

PSI
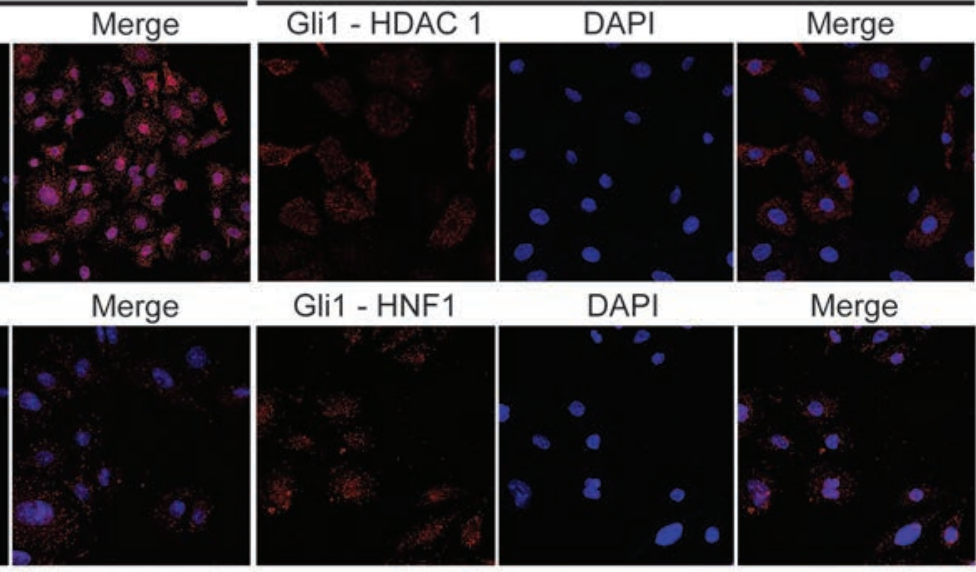

Gli1 - HNF1

DAPI

Merge
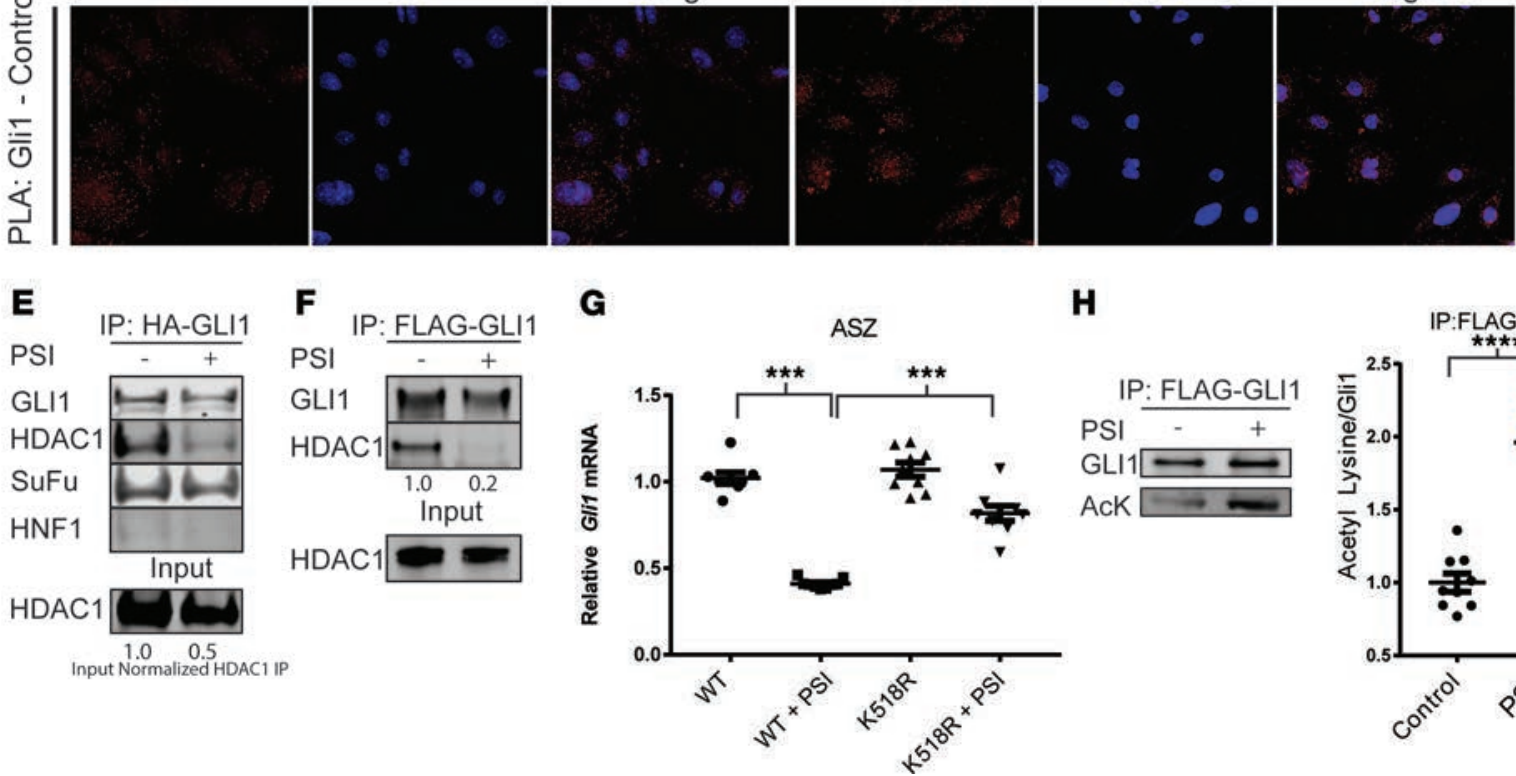

H
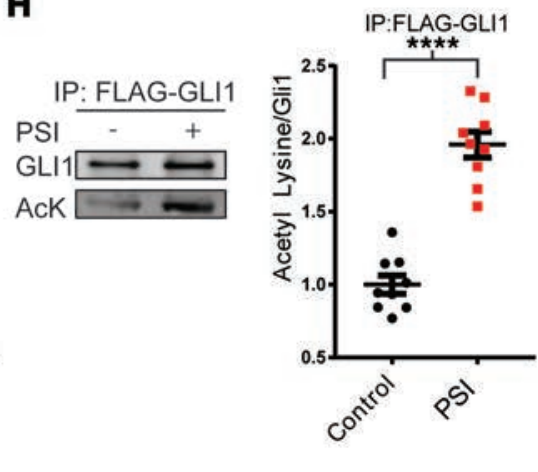

Figure 2. aPKC promotes HDAC1 recruitment and deacetylation of GLI1. (A) Comparison of LINCS data and PSI RNA-sequencing data demonstrates a strong overlap in expression fold change between aPKC inhibitor (PSI) and HDAC inhibitors. ASZ cells were transfected with (B) HDAC1 ( $n=3$; 2-way ANOVA) or (C) p300 ( $n=3$, 2-way ANOVA), and continuous cell growth was measured using Real-Time Glo reagent. The indicated cells were treated with PSI $(2.5 \mu \mathrm{M})$ after 24 hours, as indicated by the dotted line. (D) Proximity ligation assay between GLI1-HDAC1 and GLI1-HNF1 (control) in ASZ cells (original magnification, $\times 64$ confocal images). Immunoprecipitation of epitope-tagged GLI1 overexpressed in HEK $293 \mathrm{TT}$ cells (E) and ASZ cells (F), probing for epitope (HA or FLAC), HDAC1, SuFu, and HNF1. Input normalized HDAC1 IP = HDAC1 IP/HDAC1 input (E). (G) Gli1 mRNA measured by qPCR 24 hours after transfection and serum withdrawal in ASZ cells, normalized against GAPDH. WT cells versus K518R-expressing cells treated with PSI ( $n=3$, ANOVA). (H) GLI1 acetylation assayed by immunoprecipitation of FLAG-GLI1, followed by acetyl lysine and GLI1 immunoblot in ASZ cells ( $n=9$, Student's $t$ test). All control measurements are black, while PSI treatment measurements are red. Error bars represent SEM. AcK, acetylated lysine antibody. ${ }^{*} P<0.05,{ }^{* * *} P<0.001,{ }^{* * *} P<0.0001$.

Combined inhibition of HDAC1 and aPKC robustly inhibits BCC growth. As aPKC and HDAC1 act sequentially to activate GLI1 and both form positive feedback loops with GLI1 $(8,23)$, we reasoned that inhibition of both would decrease the capacity of either positive feedback loop to rescue cells from pharmacological blockade (Figure 3A). Clinically, this would decrease the effective doses, both improving efficacy and broadening the effective range for vorinostat. Indeed, combination therapy of vorinostat or parthenolide with PSI resulted in increased suppression of GLI1 protein levels at lower doses of vorinostat (Figure 3B). Consistent with a linear pathway, high-dose treatments of vorinostat 
alone converged with combination therapy vorinostat and PSI in suppressing Gli1 mRNA (Figure 3C and Supplemental Figure 14) in BCC cells. The combinatorial effect of vorinostat or parthenolide with PSI displayed a significant reduction of ASZ cell viability (Figure 3, D and E) at doses low enough to leave keratinocyte growth unaffected (Supplemental Figure 15), revealing a putative therapeutic window. We assessed the combination strategy in vivo by treating BCC allografts with subthreshold drug doses. Tumor-bearing mice were treated with half the previous dose of vorinostat $(50 \mathrm{mg} / \mathrm{kg}$ twice a day) in combination with subthreshold topical PSI administration. Notably, the combination therapy outpaced monotherapy in inhibiting the $\mathrm{HH}$ pathway in vivo (Figure $3 \mathrm{~F}$ ), which corresponded with improved outcomes in terms of tumor size (Figure 3G). Mice receiving combination therapy demonstrated no observable adverse effects, changes in weight (Supplemental Figure 16), or further hematological abnormalities (Supplemental Table 7) compared with monotherapy.

To determine the efficacy of this pharmacological blockade on disruption of the core set of genetic drivers of BCC, we assessed its effect on the BCC signature (Figure 1, A and B). Using the RNA-sequencing data from murine tumors treated with PSI and vorinostat (Figure $1 \mathrm{~J}$ ), we observed a corrective gene expression signature following both vorinostat and PSI treatment (Figure $3 \mathrm{H})$. This indicates that the treatment strategy effectively targets the majority of the molecular drivers of BCC.

We confirmed the efficacy of this treatment strategy on human disease with the use of a human BCC cell line and patient-derived BCC explants. Combination treatment on a human BCC cell line (19) (UW-BCC1) outpaced monotherapy of equivalent doses in terms of cell death, consistent with our murine BCC cell line (Supplemental Figure 17). However, cell lines only approximate human disease. Therefore, we collected primary BCCs from patients undergoing Mohs surgical operations and cultured them ex vivo for 6 hours while treating the samples pharmacologically. PSI, a myristoylated peptide, has poor tissue penetrance properties. To enhance the tissue penetrance of PSI, we fused it to a "TAT" delivery peptide (Supplemental Figure 18 and refs. 24, 25). TAT-PSI behaved similarly to PSI in terms of BCC cell line viability and aPKC activity assayed by phospho-MARK2 (Supplemental Figure 18). Remarkably, combination treatment with vorinostat and TAT-PSI resulted in a $>85 \%$ reduction in $\mathrm{HH}$ target gene abundance in these patient tumors (Figure 3I).

Development of a small-molecule aPKC inhibitor makes nuclear GLI targetable. Because peptide-based inhibitors of aPKC, such as PSI, are not clinically useful at this time, we sought to identify a novel small-molecule inhibitor of $\mathrm{PKCl}$ with suitable pharmacokinetic properties for clinical application. CRT0329868 is an azaquinazoline ATP-competitive $\mathrm{PKCl}_{1}$ inhibitor that was derived from the previously reported $\mathrm{PKC}$ inhibitor CRT0066854 through structure-based design. Biochemical PKCı enzymatic inhibitory activity was confirmed using an IMAP fluorescence polarization (FP) progressive binding assay against recombinant $\mathrm{aPKC} 1$ to determine a biochemical $\mathrm{IC}_{50}$ of $8.4 \mathrm{nM}$ on $\mathrm{PKCl}$ (Figure $4 \mathrm{~A}$ and Supplemental Figure 19), which is a $>10$-fold improvement over the first-generation ATP-competitive inhibitor CRT0066854 ( $\mathrm{IC}_{50} 86 \mathrm{nM}$ ) (7). To obtain a direct readout of cellular PKC1 activity, we used a previously reported proximal phosphorylation assay expressing a constitutively active mutant of $\mathrm{PKC}$ and FLAG-LLGL2 (7). The cellular $\mathrm{IC}_{50}$ was $34 \mathrm{nM}$, as assayed by ELISA of LLGL2 S650/654 phosphorylation (Figure 4A and Supplemental Figure 20), again over an order of magnitude improvement over CRT0066854 ( $\mathrm{IC}_{50} 870 \mathrm{nM}$ ). To determine kinase selectivity, an expanded selectivity assessment was performed at $>100$-fold the biochemical $\mathrm{IC}_{50}(1 \mu \mathrm{M})$ against a diverse panel of 71 lipid and protein kinase targets. We found that CRT0329868 was highly specific to aPKCl, with a selectivity score of $4.2 \%$ (Figure 4, A and B). Selectivity scores were calculated as the kinases with $\%$ inhibition relative to control $>90 \%$ /number of kinases tested at a test concentration of $1 \mu \mathrm{M}$. CRT0329868 also demonstrated favorable pharmacokinetic properties, with an oral bioavailability of $99 \%$, a moderate plasma half-life $\left(t_{1 / 2}=1.2 \mathrm{~h}\right)$, moderate plasma clearance $(67 \mathrm{ml} / \mathrm{min} / \mathrm{kg})$, and moderate volume of distribution $(6.51 /$ $\mathrm{kg}$ ) when dosed at $1 \mathrm{mg} / \mathrm{kg}$ i.v. and $10 \mathrm{mg} / \mathrm{kg}$ p.o. to CD-1 nude mice. Taken together, CRT0329868 represents a clinically viable aPKC inhibitor that could be implemented in BCC.

We determined the efficacy of CRT0329868 in BCC by assaying its effect on BCC cell viability and $\mathrm{HH}$ target gene transcription. We assayed the effect of CRT0329868 on the viability of our BCC cell lines. A dose-dependent response was observed with near complete cell death after 1 day of treatment at $10 \mu \mathrm{M}$ in all cell lines (Figure 4C). We observed a dose-dependent decrease in HH target gene transcript in ASZ cells, with near complete inhibition at $5 \mu \mathrm{M}$ (Figure 4D).

Finally, we assess if CRT0329868 can be used in combination with vorinostat to extend vorinostat's 

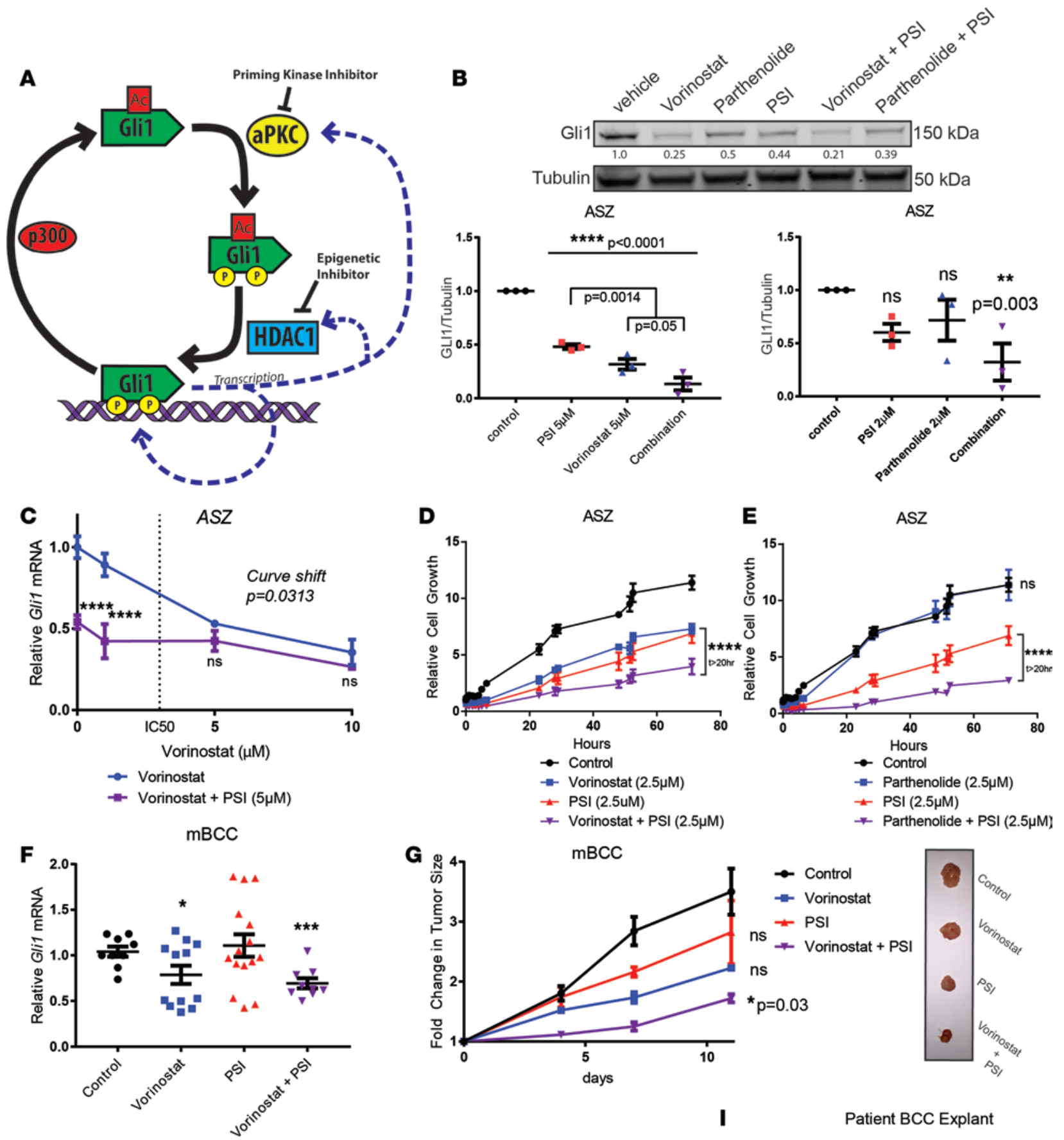

H

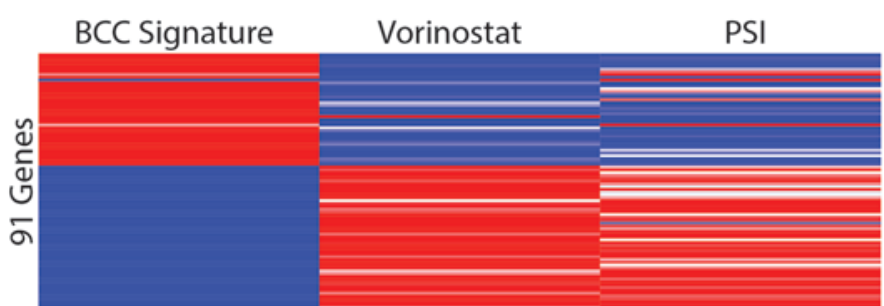

Relative Min

Relative Max

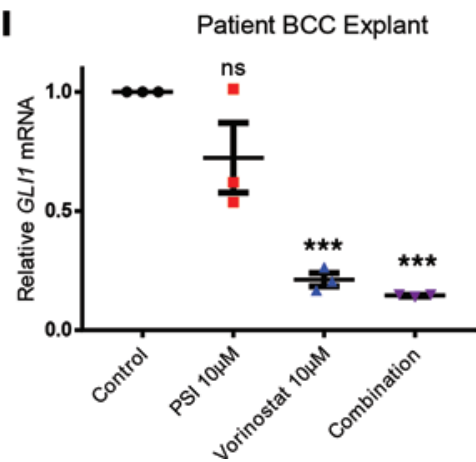

Figure 3. aPKC inhibition complements HDAC inhibition in vitro and in vivo. (A) Schematic of combination therapy rationale. (B) Western blot of Gli1 from ASZ cells, following drug treatment and serum withdrawal. Replicates are quantified after normalization against tubulin ( $n=3$ ). Additional $P$ values (ANOVA) provided for vorinostat versus combination therapy quantify differences. (C) qRT-PCR of Gli1 mRNA normalized to HPRT1 in ASZ cells following drug treatment ( $n=3$; 2-way ANOVA and linear regression). ( $\mathbf{D}$ and $\mathbf{E}$ ) ASZ cell growth following drug treatment, as measured by 
Real-Time Glo reagent ( $n=3$; 2-way ANOVA). (F) qRT-PCR of Gli1 mRNA from mouse BCC ( $n=4$; ANOVA). (G) Tumors size following drug treatment and representative images $(n=4$; ANOVA). (H) Changes to BCC signature genes (Supplemental Table 2$)$ in mouse BCC allografts following vorinostat $(n=2)$ or PSI $(n=1)$ treatment compared with control $(n=2)$. Relative values illustrated as a range from blue (upregulated) to red (downregulated). (I) qRT-PCR of Gli1 mRNA normalized to HPRT1 from patient-derived BCC explants ( $n=3$, technical replicates). All control measurements are black, HDAC inhibitor treatment measurements are blue, PSI treatment measurements are red, and combination PSI plus HDAC inhibitor treatment measurements are purple. Error bars represent SEM. ${ }^{*} P<0.05,{ }^{* *} P<0.01,{ }^{* * *} P<0.001,{ }^{* * * *} P<0.0001$.

therapeutic window. Vorinostat shifted the CRT0329868 GLI inhibition curve, demonstrating an additive effect at low doses (Figure 4D). Patient-derived BCC explants were cultured ex vivo and subsequently treated with vorinostat and CRT0329868. After 24 hours, monotherapies produced significant reductions in GLI1 RNA, but combination treatment produced the greatest reduction in $\mathrm{HH}$ output (Figure 4E). Taken together, CRT0329868 makes targeting the aPKC-HDAC1 axis in BCCs clinically viable.

\section{Discussion}

Here, we demonstrate the potential of computational drug repositioning to identify combination therapies for BCCs. Such an approach represents a rational step-wise genome-wide effort to broaden the therapeutic windows for existing drugs through combination testing, allowing them to have more diverse clinical efficacy and usage. An additional benefit is the elucidation of drug mechanisms, which will lead to additional drug targets. Our study identifies aPKC as a priming kinase for the deacetylation of GLI1 by HDAC1, providing unexpected insights into GLI1 transcription factor nuclear maturation. The development of a small-molecule inhibitor of aPKC moves this study from the theoretical to the realm of clinical possibility. Our work demonstrates in vitro and in vivo that the pharmacological inhibition of a priming kinase in combination with the use of epigenetic inhibitors is an effective strategy to increase their therapeutic window. Pharmacological blockade of the aPKC-HDAC1 axis in BCCs represents a robust strategy to inhibit $\mathrm{HH}$ activity in the nucleus, leaving fewer avenues for drug resistance to develop.

\section{Methods}

Gene expression profiling. Processed gene expression data from sporadic early-stage BCCs and normal skin was obtained from the Gene Expression Omnibus (GEO GSE6520). The Limma package from R Bioconductor was used to analyze differentially expressed genes in 23 BCCs versus 8 normal skin controls $(P \leq 0.05)$ (Supplemental Figure 2 and Supplemental Table 2).

For advanced BCCs, total RNA was isolated from advanced BCCs (6) and normal skin biopsies (5). cDNA was prepared with the Ovation RNA-Seq System (NuGEN) followed by sequencing on an Illumina 2500 platform as previously described (2). Sequencing data has been deposited in GEO (GSE58377). Tophat was used to map the raw data to the human genome (hg19) and to quantify the counts per gene. The count data were used to perform differential expression analysis using the R package, DESeq. 138 transcripts with an adjusted $P<0.05$ were considered differentially expressed (Supplemental Figure 1 and Supplemental Table 1).

Computational repositioning for drug discovery. To discover potential new therapeutic agents for advanced $\mathrm{BCC}$, we applied a systematic drug repositioning bioinformatics approach, querying a large compendium of gene expression profiles to identify candidate FDA-approved drugs. This approach ranks potential drug therapies based on how strongly the drug's gene expression signature opposes the disease signature. This method produces a negative score when the drug signature is oppositional to the disease signature and a positive score when they are concordant. This algorithm was developed Sirota et al. (15) and has been used successfully to identify novel therapeutics for small-cell lung cancer and inflammatory bowel disease.

Each gene signature from advanced BCCs and sporadic BCCs was independently integrated with gene expression data from US FDA-approved and investigational drugs contained in LINCS to identify a list of potential therapeutic targets. The LINCS database was downloaded as of September 2013 (26). The database included 66,510 high-quality gene expression profiles consisting of 12,442 distinct compounds (including 1,121 FDA-approved/investigational drugs.) In LINCS, 978 landmark genes were profiled, and the expression of the remaining genome was imputed. In our previous study, we found that using expression of landmark genes had a better performance in correlating chemical structure and gene expression that using imputed expression (26). We thus decided to use landmark genes only in the drug prediction. Further details on the computational method are detailed elsewhere (15). In total 16 candidate drugs were predicted for early BCCs and 29 for advanced BCCs $(P<0.001)$ (Supplemental Tables 3 and 4). Landmark genes involved in the 
A $\quad$ CRT00329868<smiles>C[14CH2]c1nccc(-c2nc(N3CCNCC3)c3c(C4CC4)cncc3n2)c2c3ncc(cccc1-2)[nH]3</smiles>

B

\section{Biochemical IC $\mathrm{IC}_{50}=8.4 \mathrm{nM}$}

Cellular biochemical IC $\mathrm{C}_{50}=34 \mathrm{nM}$ for inhibition of LLGL2 phosphorylation in H460 cells Kinase selectivity (71 kinases) S(90) @ $1 \mathrm{uM}=4.2 \%$

$\mathrm{Nu} / \mathrm{Nu}$ Mouse PK F = 99\%, T1/2 1.1h, CL $67 \mathrm{~mL} / \mathrm{min} / \mathrm{kg}, \mathrm{Vd} 6.5 \mathrm{~L} / \mathrm{kg}$ c
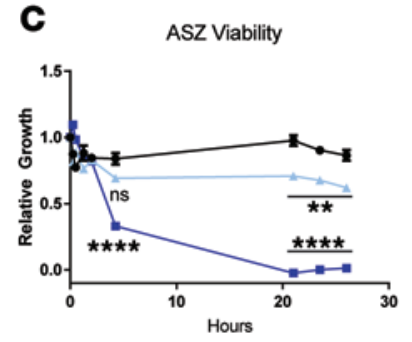

$\rightarrow$ Control
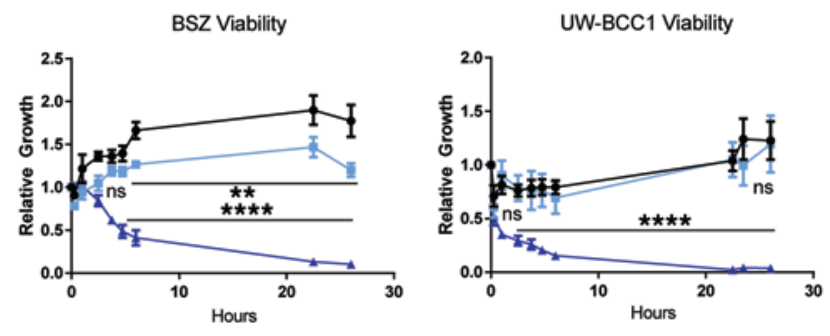

- $=$ CRT $03298681 \mu \mathrm{M} \quad$ † CRT $032986810 \mu \mathrm{M}$
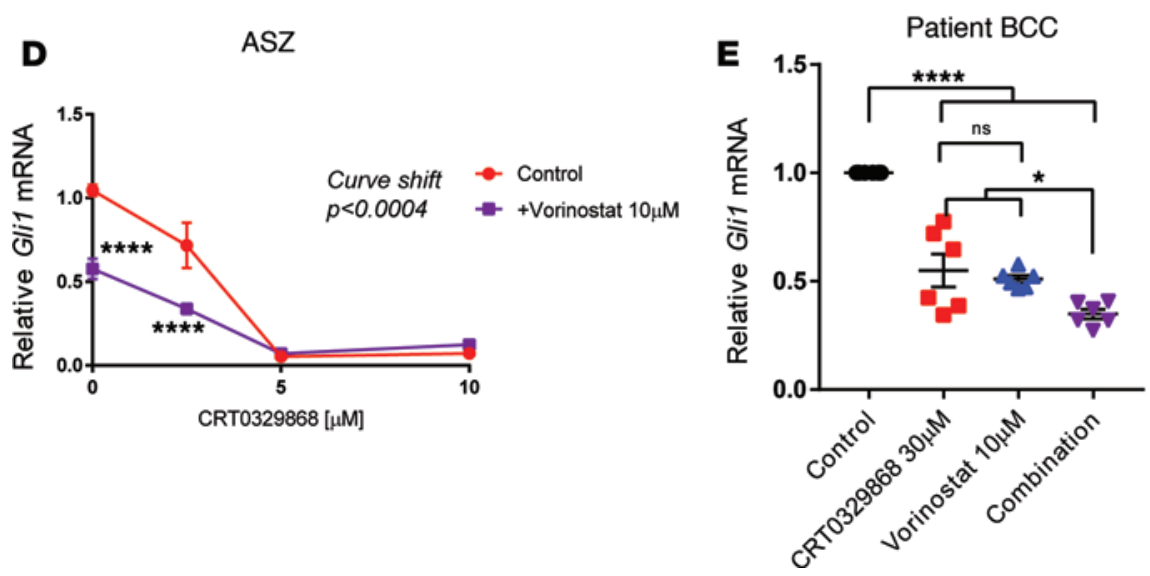

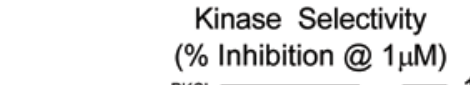

(\% Inhibition @ $1 \mu \mathrm{M}$ )

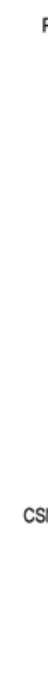

Figure 4. aPKC inhibitor CRT00329868 effectively targets aPKC BCC. (A) Structure, molecular weight, and molecular characteristics of CRT00329868. Details are included in Supplemental Figures 19-21. (B) In vitro kinase panel (71 kinases) inhibition at $1 \mu$ M. Inhibition is displayed in blue, while activation is displayed in red ( $n=3$; 2-way ANOVA). (C) Cell viability of 3 BCC cell lines in response to CRT00329868 ( $n=3$; 2 -way ANOVA). (D) Dose-response curve in ASZ cells to CRT00329868 alone or in combination with vorinostat, as assayed by Cli1 normalized to HPRT1 by qRT-PCR ( $n=9 ; 2$-way ANOVA and linear regression). (E) Primary human BCCs cultured ex vivo with indicated treatments and assayed by qRT-PCR for GL11 and HPRT1 ( $n=6$ total replicates representing 3 technical replicates of 2 biological replicates; ANOVA). Error bars represent SEM. ${ }^{*} P<0.05,{ }^{* *} P<0.01,{ }^{* * *} P<0.0001$ by ANOVA.

prediction are displayed (Supplemental Figures 3 and 4). We intersected the predicted therapeutics to identify drugs candidates that would treat both early-stage and advanced BCCs. The drugs, vorinostat, geldanamycin, and isoliquiritigenin, were predicted to treat both early-stage and advanced BCC (Supplemental Figure 5).

Prediction of combination therapies. To determine transcripts altered by PSI treatment, we applied the R package DESeq to perform differential gene expression analysis of RNA-sequencing data from duplicates of ASZ cells treated with PSI as compared with DMSO-treated controls as previously described (3). To identify chemical and genetic modulators that are correlated with PSI treatment, we identified a set of 3,171 transcripts altered with PSI treatment as compared with DMSO-treated controls (Supplemental Table 5). This signature was used to query against expression data from all 3,094 chemical compound perturbagens in the LINCS data set.

Whole-genome expression profiles were inferred from changes in the expression of 1,000 landmark genes as previously described (27). Changes in gene expression resulting from each of the perturbations were rank ordered from highest to lowest according to their differential expression relative to control treatments. Changes 
in gene expression caused by the PSI signature were compared with the cataloged expression profiles. Profiles that are positively correlated with the Query Signature were given a positive score, whereas profiles that were negatively correlated to the Query Signature were given a negative score (Supplemental Table 6). A connectivity score (mean_rankpt_2) was assigned to each of the expression profiles basis of a weighted Kolmogorov-Smirnov statistic as previously described (27).

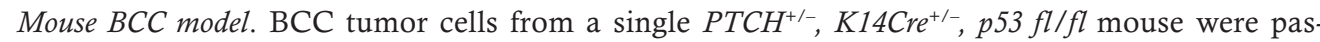
saged and suspended in Matrigel (Fisher 354234). Cells were injected subcutaneously in 2 sites on immunodeficient NOD/SCID mice, with $2 \times 10^{6}$ cells per site at 9 weeks of age. Two mice ( 4 tumors) were enrolled in each treatment arm (control, PSI only, vorinostat only, and PSI plus vorinostat). Treatments were administered twice daily, 5 times a week over 14 days. Vorinostat was administered by i.p. injection in DMSO at $50 \mathrm{mg} / \mathrm{kg}$, and PSI was administered topically in DMSO at $0.8 \mathrm{mg} / \mathrm{kg}$. Solutions were divided into single-use aliquots and stored at $-20^{\circ} \mathrm{C}$.

Antibodies. The following antibodies were used: GLI1 (Cell Signaling, 2534S [immunoblotting]; R\&D, AF3455 [immunofluorescence]; Santa Cruz, sc-6152 [immunoprecipitation]; Developmental Studies Hybridoma Bank, PCRP-Gli1-1E6 [immunoblotting]); Tubulin (Developmental Studies Hybridoma Bank, E7-c); HDAC1 (Santa Cruz, sc-7872); SuFu (Abcam, ab28083); HNF1 (Santa Cruz, sc-8986); acetylated-lysine (Cell Signaling, 9441S); and anti-MARK2 (phospho T595) antibody (Abcam, ab34751).

Drugs. The following drugs were used: vorinostat (APEX BIO, A4084); parthenolide (Santa Cruz, sc-3523); PSI (Tocris, 2549).

Quantitative PCR. RNA was isolated using Qiagen's RNeasy Mini Kit according to the manufacturer's protocols. The TaqMan RNA-to-CT 1-Step Kit (Thermo Fisher) was used for reverse transcription and quantitative PCR. TaqMan Gene Expression Assay Probes (Life Technologies, GLI1; FAM-MGB probe Mm00494654_m1) and Hprt (Mm01545399_m1) were used in an assay-dependent concentration.

Cell culture. ASZ001 (ASZ) BCC cells were cultured in 154CF media (Life Technologies) supplemented with $2 \%$ chelated fetal bovine serum, Human Keratinocyte Growth Supplement (Thermo Fisher), Penn-Strep, and $0.05 \mathrm{mM} \mathrm{CaCl}_{2}$. Experiments assaying $\mathrm{HH}$ signaling were carried out in serum-free conditions. NIH-3T3, HaCaT, and HEK-293T cells were cultured in DMEM supplemented with 10\% fetal bovine serum. Unless otherwise specified, PSI was administered at $5 \mu \mathrm{M}$. NIH-3T3, HaCaT, and HEK293T cells were obtained from ATCC, and ASZ001 cells were a gift from the lab of Ervin Epstein.

Mammalian cell transfection was performed using FuGENE 6 Transfection Reagent (Promega), Lipofectamine LTX with Plus Reagent (Thermo Fisher), and the MEF 1 Nucleofector Kit (Lonza) per the manufacturer's protocols. Transient transfection mammalian expression vectors included pCS2HA and pCS2FLAG. Stable expression produced by lentiviral vector pLEX infection was supplemented with Polybrene Reagent (Millipore).

Viability assays were performed using the RealTime-Glo MT Cell Viability Assay (Promega) following the manufacturer's protocol. Luminescence was measured by the SpectraMax M5 plate reader (Molecular Devices) at $37^{\circ} \mathrm{C}$ over a 1.5 -second interval.

We conducted regular PCR-based mycoplasmic contamination tests and disposed of any contaminated cells.

Immunoblotting. Whole-cell extracts were harvested using radioimmunoprecipitation (RIPA) buffer supplemented with protease and phosphatase inhibitors (Roche) and run on gradient SDS-PAGE gels (Life Technologies), followed by being transferred onto nitrocellulose membranes. Primary antibodies were used in an assay-dependent concentration in 5\% Bovine Serum Albumin in TBST. Fluorescent secondary antibodies compatible with Odyssey CLx (Licor) were used for imaging.

Fractionation. To prepare nuclear extracts, cells were resuspended in hypotonic lysis buffer $(10 \mathrm{mM}$ HEPES, pH7.9, $1.5 \mathrm{mM} \mathrm{MgCl}_{2}$, and $10 \mathrm{mM} \mathrm{KCl}$ ) and Dounce homogenized. Isolated nuclei were pelleted and resuspended in RIPA buffer.

Immunoprecipitation. Cell lysates were prepared in 1\% Triton X-100 in TBS supplemented with protease and phosphatase inhibitors (Roche). Anti-FLAG M2 Magnetic Beads (MilliporeSigma) and Pierce AntiHA Magnetic Beads (Thermo Fisher) were preblocked in 5\% BSA prior to immunoprecipitation. Washes were carried out at room temperature in lysis buffer.

Immunofluorescence. Cells were fixed in 4\% formaldehyde in PBS and prepared per a general immunofluorescence protocol (abcam). Primary antibodies were used at the manufacturers' recommended concentration (1:500, DSHB, E7). We used the following fluorescent-labeled secondary antibodies: Alexa Fluor 488, Alexa 
Fluor 555, Alexa Fluor 594, and Alexa Fluor 647 (1:500, Life Technologies). Confocal imaging was carried out using a Leica SP8 microscope equipped with an adjustable white light laser and hybrid detectors. Proximity ligation assays were conducted per the manufacturer's protocol (Duolink/MilliporeSigma).

Human explant. Central biopsies of clinically diagnosed BCC tumors from Mohs surgery patients at the Stanford Dermatology Clinic were quartered and cultured in EpiLife Media (Life Technologies) plus $\mathrm{CaCl}_{2}$ for 6 hours, prior to RNA extraction.

Biochemical kinase assay. The ability of compounds to inhibit the kinase activity of recombinant human baculovirus-expressed full-length PKCl was measured using the IMAP FP progressive binding system (Molecular Devices R8127) in 384-well black, nonbinding, flat-bottom assay plates (Corning 3575). The assay mixture (final volume $=10 \mu \mathrm{l}$ ) contained $20 \mathrm{mM}$ Tris-HCL ( $\mathrm{pH} 7.5$ ), $150 \mu \mathrm{M}$ ATP, $10 \mathrm{mM} \mathrm{MgCl}, 0.01 \%$ Triton X-100, $250 \mu \mathrm{M}$ EGTA, 1 mM DTT, 15 pM PKCl (EMD Millipore 14-505), 100 nM FAM-PKC $\varepsilon$-pseudosubstrate (Molecular Devices RP7548), 0.1\% DMSO, and various concentrations of test compounds. Compound dilutions (prepared in 100\% DMSO) were added to the assay plate at $100 \mathrm{nl}$ using the BioMek NX pin tool (Beckman Coulter). Enzyme reactions were initiated by the addition of ATP (MilliporeSigma A7699), followed by incubation of the plates for 1 hour in a $25^{\circ} \mathrm{C}$ incubator. A $20-\mu$ aliquot of IMAP detection reagent (1:400 in 85\% 1X Binding Buffer A and 15\% 1X Binding Buffer B) was added to each well, followed by a 2-hour incubation at $25^{\circ} \mathrm{C}$. FP was then measured using the PerkinElmer Envision 2102 multi-label plate reader (PerkinElmer) using the FP dual mirror, FP480 excitation filter, and P-pol 535 and S-pol 535 emission filters. Data analysis was performed using ActivityBase (IDBS). $\mathrm{IC}_{50}$ values were calculated by plotting the percentage inhibition versus $\log _{10}$ of the concentration of the compound and fitting to the 4-parameter logistic model (top and bottom constrained to 100 and 0, respectively) in XLFit 4 (IDBS)

Kinase specificity assay. The Invitrogen SelectScreen Biochemical Kinase Profiling Service using the Z'-LYTE Screening Assay was used against a panel of 71 kinases at a test concentration of $1 \mu \mathrm{M}$.Selectivity score were calculated as kinases inhibited $>90 \%$ divided by total kinases assayed. CRT0329868 demonstrated a high degree of selectivity in this panel, with a selectivity score of 0.042 .

RNA sequencing. RNA was isolated from mouse BCC allografts using the Qiagen RNeasy Mini Kit according to the manufacturer's protocols. Library preparation and sequencing was performed by the Stanford Genomics Core Facility.

Development of CRT0329868. CRT0329868 was developed by JR using structure-based design as reported previously (28). CRT0329868 is referred to as compound 1253 in the patent. At time of publication, CRT0329868 may be obtained through an MTA from Cancer Research Technology (JR).

siRNA. Predesigned MilliporeSigma MISSION siRNAs against selected targets were transfected using Lipofectamine RNAiMax reagent. The following siRNA products from Sigma were used: HDAC1 (SASI Mm02_00313417, SASI_Mm01_00159502, SASI_Mm01_00159497), HDAC2 (SASI_Mm01_00100698, SASI_Mm01_00100699, SASI_Mm01_00100700), and aPKC iota (SASI_Mm01_00134911, SASI_ Mm01_00134912,SASI_Mm01_00134913).

Statistics. Significance was determined by 2-tailed $t$ test or ANOVA, as indicated in the figure legends. A $P$ value of less than 0.05 was used to determine significance.

Study approval. This study was approved by the Stanford University Institutional Review Board (protocol 18325), with a waiver of consent from participants.

\section{Author contributions}

ANM, SXA, ASB, NMU, and AEO designed the cell-based experiments. ANM, KYS, and AEO wrote the manuscript. ANM performed the majority of the experiments. KYS, MS, and BC designed and performed the computational analysis. JR, GRO, MAA, BDD, BRR, and CAZ designed and developed the CRT0329868 compound. JYT, KYS, and AEO designed the mouse experiments. MAF and AL performed animal experiments under the guidance of JYT, KYS, and AEO, who jointly supervised the study. SZA and $\mathrm{TH}$ assisted in procurement of human BCC explants. AB assisted KYS, MS, and BC in the computational analysis. All authors critically reviewed the manuscript.

\section{Acknowledgments}

We would like to acknowledge Lisa D. Aimone for her contributions to the pharmacokinetic analysis of CRT0329868. The work was funded by RO1AR04786 and R01ARO54780 (AEO), R00CA176847 (SXA), and the American Skin Association (KS). 
Address correspondence to: Kavita Y. Sarin or Anthony E. Oro, Program in Epithelial Biology and Department of Dermatology, 269 Campus Drive CCSR 2145, Stanford University School of Medicine, Stanford, California 94305, USA. Phone: 650.804.2899; Email: ksarin@stanford.edu (K.Y. Sarin). Phone: 650.725.7022; Email: oro@stanford.edu (A.E. Oro).

S.X. Atwood's present address is: Department of Developmental and Cell Biology, University of California, Irvine, Irvine, California, USA.

1. Chang AL, Oro AE. Initial assessment of tumor regrowth after vismodegib in advanced basal cell carcinoma. Arch Dermatol. 2012;148(11):1324-1325.

2. Atwood SX, et al. Smoothened variants explain the majority of drug resistance in basal cell carcinoma. Cancer Cell. 2015;27(3):342-353.

3. Atwood SX, Li M, Lee A, Tang JY, Oro AE. GLI activation by atypical protein kinase $\mathrm{C} 1 / \lambda$ regulates the growth of basal cell carcinomas. Nature. 2013;494(7438):484-488

4. Hutchin ME, et al. Sustained Hedgehog signaling is required for basal cell carcinoma proliferation and survival: conditional skin tumorigenesis recapitulates the hair growth cycle. Genes Dev. 2005;19(2):214-223.

5. Sekulic A, et al. Efficacy and safety of vismodegib in advanced basal-cell carcinoma. N Engl J Med. 2012;366(23):2171-2179.

6. Erdogan E, et al. Aurothiomalate inhibits transformed growth by targeting the PB1 domain of protein kinase Ciota. J Biol Chem. 2006;281(38):28450-28459.

7. Kjær S, et al. Adenosine-binding motif mimicry and cellular effects of a thieno[2,3-d]pyrimidine-based chemical inhibitor of atypical protein kinase C isoenzymes. Biochem J. 2013;451(2):329-342.

8. Canettieri G, et al. Histone deacetylase and Cullin3-REN(KCTD11) ubiquitin ligase interplay regulates Hedgehog signalling through Gli acetylation. Nat Cell Biol. 2010;12(2):132-142.

9. Coni S, et al. Selective targeting of HDAC1/2 elicits anticancer effects through Gli1 acetylation in preclinical models of SHH Medulloblastoma. Sci Rep. 2017;7:44079.

10. Zhao J, Quan H, Xie C, Lou L. NL-103, a novel dual-targeted inhibitor of histone deacetylases and hedgehog pathway, effectively overcomes vismodegib resistance conferred by Smo mutations. Pharmacol Res Perspect. 2014;2(3):e00043.

11. Coni S, et al. Gli2 acetylation at lysine 757 regulates hedgehog-dependent transcriptional output by preventing its promoter occupancy. PLoS One. 2013;8(6):e65718.

12. Falkenberg KJ, Johnstone RW. Histone deacetylases and their inhibitors in cancer, neurological diseases and immune disorders. Nat Rev Drug Discov. 2014;13(9):673-691.

13. Mann BS, et al. Vorinostat for treatment of cutaneous manifestations of advanced primary cutaneous T-cell lymphoma. Clin Cancer Res. 2007;13(8):2318-2322.

14. Hoelder S, Clarke PA, Workman P. Discovery of small molecule cancer drugs: successes, challenges and opportunities. Mol Oncol. 2012;6(2):155-176.

15. Sirota $\mathrm{M}$, et al. Discovery and preclinical validation of drug indications using compendia of public gene expression data. $S c i$ Transl Med. 2011;3(96):96ra77.

16. Jahchan NS, et al. A drug repositioning approach identifies tricyclic antidepressants as inhibitors of small cell lung cancer and other neuroendocrine tumors. Cancer Discov. 2013;3(12):1364-1377.

17. Dudley JT, et al. Computational repositioning of the anticonvulsant topiramate for inflammatory bowel disease. Sci Transl Med. 2011;3(96):96ra76.

18. Cho HG, Fiorentino D, Lewis M, Sirota M, Sarin KY. Identification of $\alpha$-adrenergic agonists as potential therapeutic agents for dermatomyositis through drug-repurposing using public expression datasets. J Invest Dermatol. 2016;136(7):1517-1520

19. Noubissi FK, et al. Role of CRD-BP in the growth of human basal cell carcinoma cells. J Invest Dermatol. 2014;134(6):1718-1724.

20. Wang GY, So PL, Wang L, Libove E, Wang J, Epstein EH. Establishment of murine basal cell carcinoma allografts: a potential model for preclinical drug testing and for molecular analysis. J Invest Dermatol. 2011;131(11):2298-2305.

21. So PL, et al. Long-term establishment, characterization and manipulation of cell lines from mouse basal cell carcinoma tumors. Exp Dermatol. 2006;15(9):742-750.

22. Gopal YN, Arora TS, Van Dyke MW. Parthenolide specifically depletes histone deacetylase 1 protein and induces cell death through ataxia telangiectasia mutated. Chem Biol. 2007;14(7):813-823.

23. Atwood SX, Li M, Lee A, Tang JY, Oro AE. GLI activation by atypical protein kinase $\mathrm{C} 1 / \lambda$ regulates the growth of basal cell carcinomas. Nature. 2013;494(7438):484-488

24. Xia H, Mao Q, Davidson BL. The HIV Tat protein transduction domain improves the biodistribution of $\beta$-glucuronidase expressed from recombinant viral vectors. Nat Biotechnol. 2001;19(7):640-644.

25. Schwarze SR, Ho A, Vocero-Akbani A, Dowdy SF. In vivo protein transduction: delivery of a biologically active protein into the mouse. Science. 1999;285(5433):1569-1572.

26. Chen B, Greenside P, Paik H, Sirota M, Hadley D, Butte AJ. Relating chemical structure to cellular response: an integrative analysis of gene expression, bioactivity, and structural data across 11,000 compounds. CPT Pharmacometrics Syst Pharmacol. 2015;4(10):576-584

27. Peck D, Crawford ED, Ross KN, Stegmaier K, Golub TR, Lamb J. A method for high-throughput gene expression signature analysis. Genome Biol. 2006;7(7):R61.

28. Breslin HJ, et al., inventors; Ignyta, Inc., Cancer Research Technology Limited, assignees. Azaquinazoline inhibitors of atypical protein kinase c. US patent PCT/US2013/062085. July 16, 2015. https://www.google.com/patents/WO2014052699A9?cl=zh. Accessed October 10, 2017. 